\title{
Erratum to: Dose-response efficacy and safety of PA21 in Japanese hemodialysis patients with hyperphosphatemia: a randomized, placebo-controlled, double-blind, Phase II study
}

\author{
Fumihiko Koiwa $^{1} \cdot$ Akira Terao $^{2}$
}

Published online: 10 November 2016

(C) Japanese Society of Nephrology 2016

\section{Erratum to: Clin Exp Nephrol \\ DOI 10.1007/s10157-016-1299-z}

In the original publication of this paper, the second sentence of Fig. 3 is incorrectly published as:

The mean serum phosphorus levels were significantly higher in all the PA21 groups in all the time-points after administering the PA21 dose (all $P<0.001$, one-sample $t$ test).

The corrected sentence is given below:

The mean serum phosphorus levels were significantly lower in all the PA21 groups at all the time-points after administering the PA21 dose (all $P<0.001$, one-sample $t$ test).

The online version of the original article can be found under doi:10.1007/s10157-016-1299-z.

Fumihiko Koiwa

koiwa-f@med.showa-u.ac.jp

1 Division of Nephrology, Department of Medicine, Showa University Fujigaoka Hospital, 1-30 Fujigaoka, Aoba-ku, Yokohama 227-8501, Japan

2 Biostatistics, Faculty of Pharmaceutical Sciences, Josai University, Sakado, Japan 